trauma, the opportunity to piece together what happened. This cannot occur in the same way if PD is used individually. Therapeutic group support factors are also missing. PD within 48 hours for primary victims may be inappropriate given that numbing and dissociative symptoms may predominate. Reactions and needs of primary and secondary victims may not evolve in the same way.

Within PD, a number of therapeutic factors may operate: social support, information processing, facilitation of constructive coping and education about the stress response syndrome. Which factor or combination of factors are necessary to prevent PTSD?

Distinction must be made in relation to the nature of the trauma. PD may be useful after exposure to a single circumscribed event. It may not be appropriate as a single 'end of trauma' session after exposure to an event/s which has been ongoing over a period of weeks. For example, no benefit from the technique was apparent in a controlled study conducted in soldier body handlers who had also been traumatised over a prolonged period during the Gulf conflict and who were offered a single PD session some weeks after traumatic exposure (Deahl et al, 1994). In contrast, in an important study not mentioned by Bisson \& Deahl, Alexander (1993) reported that police body handlers who received interventions including daily PD showed no increase in psychiatric morbidity three years later. Ongoing debriefing for ongoing trauma may be necessary.

Psychological debriefing is rarely considered in a theoretical context. The possibility of iatrogenic effects is overlooked. The presentation of traumatic material without allowing sufficient time for the habituation of anxiety may serve as a retraumatisation experience for some. Group studies may mask this effect.

Alexander, D. A. (1993) Stress among police body handlers. A long-term follow up. British Journal of Psychiatry, 163, 806-808.

DenkI, M. P., Gnlham, A. B., Thomas, J., et al (1994) Psychological sequelae following the Gulf War. Factors associated with subsequent morbidity and the effectiveness of psychological debriefing. British Journal of Psychiatry, 165, 60-65.

DYregov, A. (1989) Caring for helpers in disaster situations: psychological debriefing. Disaster Management, 2, 25-30.

Mrrchel L, J. T. (1983) When disaster strikes. The critical incident stress debriefing process. Journal of Emergency Medical Services, 8, 36-39.

\author{
District Clinical Psychology Service \\ Victoria Hospital \\ Swindon SNI 4JA
}

Princess Alexandra Hospital

Royal Air Force Wroughton Wiltshire SN4 OQJ

W. BusutrII

\section{Charles Bonnet syndrome}

SIR: Teunisse et al (BJP, February 1995, 166, 251253), report a large series of patients who are reported to have the Charles Bonnet syndrome emanating from an ophthalmological clinic.

The major value of the investigation of the Charles Bonnet syndrome is the light that it sheds upon the pathogenesis of visual hallucinations in the absence of other psychopathology. The definitions used in this paper are derived from the work of Gold \& Rabins (1989). Despite early authors stressing the lack of cognitive impairment in their patients neither Gold \& Rabins (1989) nor the authors of this paper include this in their definitions. Even if few of the patients fall into the putatively cognitively impaired range (as is the case in this paper) this is important to note. Holroyd et al (1994) using a telephone interview technique that does not depend on visual cues demonstrated that the hallucinators in their population have significantly lower cognitive scores $(P<0.001)$ than the non-hallucinators. Howard \& Levy (1994) demonstrated a similar finding in late paraphrenics with 'Charles Bonnet-plus' hallucinations despite excluding all with a Mini-Mental State score below 24.

Several authors record complex visual hallucinations as the precursor of dementing illnesses. Given the numerous reports of brain lesions in those with Charles Bonnet syndrome without cognitive impairment, the cognitive state of both the individual patient and that of the population from which the subject is drawn needs to be described in some detail.

The lax use of the term Charles Bonnet syndrome has been criticised (Berrios \& Brook, 1982) and should be reserved only for those who exhibit no cognitive impairment. Terms such as Charles Bonnet-plus or complex visual hallucinosis should perhaps be used when studies are being attempted across diagnostic categories or when cognitive status is not investigated in detail.

Berrios, G. E. \& Brook, P. (1982) The Charles Bonnet syndrome and visual perceptual disorders of the elderly. Age \& Ageing, 11, 17-23.

GolD, K. \& RABINS, P. V. (1989) Isolated visual hallucinations and the Charles Bonnet syndrome: $A$ review of the literature and presentation of six cases. Comprehensive Psychiatry. 30, 90-98. 
Holroyd, S., RAbins, P. V., Finkelstern, D., et al (1994) Visual hallucinations in patients from an ophthalmology clinic and medical clinic population. Journal of Nervous and Mental Disorders, 182, 273-276.

HowARD, R. \& LEVY, R. (1994) Charles Bonnet syndrome-plus: complex visual hallucinations of the Charles Bonnet type in late paraphrenia. International Journal of Geriatric Psychiatry, 9, $399-404$.

\section{The London Hospital Medical College \\ Turner Street}

London E1 2AD

C. BALL

\section{The nature of dysthymia}

SIR: While reconceptualisation of dysthymia as a 'chronic' and 'subaffective' form of major depressive disorder for which pharmacotherapy is the mainstay of treatment (BJP, February 1995, 166, 174-183) may promote the treatment of a subgroup of subjects, this proposition should be tempered as follows.

Dysthymia has been termed 'a new plastic box for some rather old wine' (Goldberg \& Bridges, 1990). It represents a highly heterogeneous group of conditions that are frequently associated with intractable interpersonal difficulties and social misfortunes - hence its considerably higher prevalence rates in poorer people. When the term is used outside of the more affluent West, it has been criticised for medicalising social problems related to severe economic, political and health constraints which create endemic feelings of hopelessness and helplessness. In these contexts, despair is a response to real conditions of chronic deprivation and persistent losses, while negative cognition is an accurate mapping of one's place in an oppressive social system (Kleinman, 1987). The evidence for considering dysthymia as an affective disorder is largely non-specific. The positive response of a subgroup of patients to thymoleptics is no exception. Although tricyclic drugs are labelled as antidepressants, they ameliorate not only depression, but also a diversity of other disease categories.

The official recommendation of 'recognising' dysthymia as a pharmaco-responsive variant of major depressive disorder runs the risk of encouraging practitioners to replace non-pharmacological ways of relieving chronic social distress with an excessive reliance on drug therapy. There is no perfect shorthand for the complex illness reality of chronically dysphoric subjects. The question of whether dysthymia is a 'sub-affective' or 'supra-neurotic' disorder is epistemological and political in nature.

GoldberG, D. P. \& Bridges, K. W. (1990) Epidemiological observations on the concept of dysthymic disorder. In Dysthy- mic Disorder (eds S. W. Burton \& H. S. Akiskal), pp. 104-111. London: Gaskell.

KLEINMAN, A. M. (1987) Anthropology and psychiatry: the role of culture in cross-cultural research on illness. British Journal of Psychiatry, 151, 447-454.

Prince of Wales Hospital

SING LeE

Shatin, N.T.

Hong Kong

\section{Where shall we put lithium et al?}

SIR: It is now 32 years since the notion of a new and special psychiatric role for lithium was first aired in this Journal (Schou, 1963). Nevertheless there is still uncertainty about the category in which this drug and its alternatives, carbamazepine, valproate, etc., should be placed and what they should be called. In textbooks and lists of 'current reading' they have appeared under tranquillisers, or antimanic drugs, or antidepressant drugs, or they have been catalogued as 'mood stabilisers'.

This confuses and misleads. Lithium and its alternatives act therapeutically on manias and depressions and prophylactically on manic and depressive recurrences. They are neither neuroleptics nor tranquillizers, and they have a wider range than drugs with exclusively anti-manic or exclusively antidepressant action. Clearly they belong in a class of their own.

But what should that class be called? The term 'mood stabilisers' is hardly a happy choice, for the drugs do not stabilise abnormal moods; they do not perpetuate a mania or 'freeze' a depression. What they do is to counteract episodes of abnormal mood and maintain normal mood in patients with recurrent manic-depressive illness.

In 1963 the terms 'normothymotics' and 'mood normalisers' were proposed. They never caught on, and the former has been spurned for being ugly and of bastard linguistic origin (Johnson, 1984). Well, the beauty or ugliness of a name is a matter of personal taste, and there is precedence for words of mixed Latin-Greek parentage. However, that particular term is not important; the crucial point is that lithium and its alternatives are placed in a special class, and I still feel that 'mood normalisers' is an apt name. But there may be other possibilities. The adjective 'euthymic' has been used for the condition of manic-depressive patients during the intervals between episodes; can it possibly function as a noun and a class name, 'euthymics'? Linguistically minded readers of the $B J P$ may have further proposals, and a discussion could perhaps lead to agreement about a fitting term. 